\title{
PERFIL EPIDEMIOLÓGICO DE MALÁRIA GRAVE EM RECÉM NASCIDOS E ADOLESCENTES ATENDIDOS EM 2016 EM UM HOSPITAL DE REFERÊNCIA DO ESTADO DO AMAPÁ, BRASIL
}

\section{ARTIGO ORIGINAL}

BRITO, Maysa Vasconcelos de ${ }^{1}$, FRANÇA, Ana Maria Braga da Silva², FECURY, Amanda Alves ${ }^{3}$, OLIVEIRA, Euzébio de ${ }^{4}$, DENDASCK, Carla Viana ${ }^{5}$, DIAS, Cláudio Alberto Gellis de Mattos ${ }^{6}$

BRITO, Maysa Vasconcelos de. Et al. Perfil epidemiológico de malária grave em recém nascidos e adolescentes atendidos em 2016 em um hospital de referência do Estado do Amapá, Brasil. Revista Científica Multidisciplinar Núcleo do Conhecimento. Ano 05, Ed. 06, Vol. 12, pp. 05-23. Junho de 2020. ISSN: 24480959, Link de acesso: https://www.nucleodoconhecimento.com.br/saude/malariagrave, DOI: 10.32749/nucleodoconhecimento.com.br/saude/malaria-grave

\section{RESUMO}

O presente estudo levou em consideração não somente as infecções por Plasmodium falciparum que comumente é preconizado como o principal causador da malária grave, mas foram cuidadosamente descritas as complicações relacionadas ao Plasmodium vivax. O objetivo deste artigo foi identificar o perfil epidemiológico, clínico e laboratorial da malária grave em pacientes atendidos no Hospital da

\footnotetext{
${ }^{1}$ Biomédica, Doutora em Doenças Topicais, Professora e pesquisadora Faculdade Estácio de Sá, Macapá.

2 Biomédica, Técnica do Hospital da Criança e do Adolescente (Macapá - Amapá).

${ }^{3}$ Biomédica, Doutora em Doenças Topicais, Professora e pesquisadora do Curso de Medicina do Campus Macapá e do Programa de Pós-Graduação em Ciências da Saúde (PPGCS), Universidade Federal do Amapá (UNIFAP).

${ }^{4}$ Biólogo, Doutor em Doenças Topicais, Professor e pesquisador do Curso de Educação Física, Universidade Federal do Pará (UFPA).

${ }^{5}$ Teóloga, Doutora em Psicanálise, pesquisadora do Centro de Pesquisa e Estudos Avançados (CEPA).

${ }^{6}$ Biólogo, Doutor em Teoria e Pesquisa do Comportamento, Professor e pesquisador do Programa de PósGraduação em Educação Profissional e Tecnológica (PROFEPT) do Instituto de Ensino Básico, Técnico e Tecnológico do Amapá (IFAP).
}

RC: 52768

Disponível em: https://www.nucleodoconhecimento.com.br/saude/malaria-grave 
Criança e do Adolescente em Macapá - Amapá. Foi realizado um estudo epidemiológico, descritivo de caráter retrospectivo. Os dados foram obtidos através de consulta em prontuários dos pacientes referente ao ano 2016. Foram considerados malária grave 47 casos. Predominaram pacientes do sexo masculino com $63,8 \%$ e da faixa etária até 5 anos com $59,6 \%$ dos casos. Quanto a espécie, destacou-se, com maior frequência as infecções por $P$. vivax com $72 \%$ em relação ao $P$. falciparum com $28 \%$ de casos. Os meses com maior número de internações foram setembro e novembro ambos com 17\%. As principais manifestações clínicas encontradas foram: febre, palidez e tosse. As condições que indicam gravidade que mais ocorreram: vômito $87 \%$, icterícia $23 \%$, dispneia e idade $<1$ ano ambas com $21 \%$. Os exames hematológicos mostraram que $91 \%$ dos pacientes apresentaram hemácias abaixo dos valores de referência e 100\% dos casos estavam com hemoglobina e hematócrito baixos; foi evidenciado plaquetopenia em $72 \%$ dos casos. Resultados alterados das dosagens bioquímicas de maior importância clínica foram: uréia, transaminases, glicimeia e proteína $C$ reativa. $A$ identificação de dados epidemiológicos, clínicos e laboratoriais da malária grave contribui para o diagnóstico precoce e o tratamento adequado da doença.

Palavras-chave: Malária grave, Plasmódio vivax, epidemiologia.

\section{INTRODUÇÃO}

A malária constitui um importante problema de saúde pública de grande interesse mundial. É uma doença infecciosa causada por protozoários do gênero Plasmodium transmitidos pela picada do mosquito fêmea do gênero Anopheles infectado. As principais espécies associadas à malária humana são: Plasmodium falciparum, $P$. vivax, P.malarie e $P$. ovale. Recentemente foram registrados alguns casos de malária a partir de infecções com $P$. knowlesi e $P$. cynomolgi, conhecidas espécies causadoras de malária em símios (TA et al., 2014; RAMASSAMI, 2014). As mais comuns e comprovadamente encontradas no Brasil são: P.vivax, $P$. falciparum e $P$.

$\mathrm{RC}: 52768$

Disponível em: https://www.nucleodoconhecimento.com.br/saude/malaria-grave 
malarie, uma vez que o P. ovale, é mais comum em regiões da África (BRASIL, 2009).

O desenvolvimento dos vetores transmissores da malária é muito viável na Amazônia legal. O estado do Amapá é um estado da região norte, componente da Amazônia Legal e um dos estados com um ambiente mais propicio à procriação do vetor e à transmissão da malária. Em relação ao Brasil, o Estado do Amapá está entre as áreas de médio e alto risco de contaminação (SANTOS; SANTOS, 2011).

A ocorrência dos casos de malária relaciona-se a vários fatores, entre eles as atividades de extrativismo dos recursos naturais, capazes de potencializar a transmissão da doença. |Nesse sentido o Amapá se apresenta como uma região que tem sua economia e sobrevivência na utilização dos recursos naturais (MACIEL; OLIVEIRA, 2014). Diante do ambiente propício e das necessidades da população de se utilizar dos recursos naturais, a infecção por malária é alta no Amapá, e os casos com maior incidência são os causados pelo $P$. vivax, (90\% dos casos) (SANTOS; SANTOS, 2011).

Evidências mostram que o espectro clínico da malária sofre influência da intensidade de transmissão, da espécie de parasita e do estado imunológico do paciente. Dessa forma, a apresentação clínica da malária parece diferir entre crianças e adultos (WASSMER et al., 2015).

\section{OBJETIVO}

Identificar o perfil epidemiológico, clínico e laboratorial de malária grave em recém nascidos e adolescentes atendidos em 2016 em um hospital de referência do estado do Amapá, Brasil.

RC: 52768

Disponível em: https://www.nucleodoconhecimento.com.br/saude/malaria-grave 


\section{MÉTODO}

Trata-se de um estudo epidemiológico descritivo de caráter retrospectivo, desenvolvido no Hospital da criança e do Adolescente (HCA) localizado na cidade de Macapá no Estado do Amapá, norte do Brasil. Os dados para esta pesquisa foram obtidos através de consulta ao banco de dados do Serviço de Arquivo Médico (SAME) do HCA. Para registro das informações coletadas foram retirados do banco de dados os seguintes tópicos: variáveis demográficas (sexo, idade, procedência...), variáveis clínicas: número de internação, tempo de internação, espécie infectante ( $P$. vivax, $P$. falciparum, P. malarie), manifestações clínicas (febre, mialgia, calafrio, oligúria, icterícia, hiperpirexia (maior que $41^{\circ} \mathrm{C}$ ), convulsões, etc) e resultados de exames laboratoriais (hemograma, hemoscopia (gota espessa) e dosagens bioquímicas (uréia, creatinina, aspartato aminotransferase (AST) e alanina aminotransferase (ALT), PCR (Proteína C Reativa) e glicemia).

Foram incluídos crianças e adolescentes de ambos os sexos (na faixa etária de 29 dias de nascido a 15 anos de idade) atendidos no período de $1^{\circ}$ de Janeiro a 31 de dezembro de 2016, com confirmação laboratorial da malária (pesquisa de plasmódio com resultados positivos) e que apresentaram manifestações clínicas e laboratoriais de malária grave de acordo com o descrito no Guia Prático de Malária do ano de 2010, que dispõe das condições indicativas de gravidade da doença e necessidade de internação: pacientes imunodeprimidos, pacientes com qualquer um dos sinais de perigo para malária grave: (hiperpirexia maior que $41^{\circ} \mathrm{C}$, convulsões, hiperparasitemia ( $>200.000 / \mathrm{mm}^{3}$ ), vômitos repetidos, oligúria, dispinéia, anemia intensa, icterícia, hemorragias e hipotenção arterial.

O do projeto de pesquisa foi submetido e aprovado pelo Comitê de Ética e Pesquisa da Faculdade Estácio de Macapá, segundo as normas éticas estabelecidas na Resolução do Conselho Nacional de Saúde № 466 de 12 de dezembro de 2012 que trata da pesquisa envolvendo seres humanos (BRASIL, 2013).

RC: 52768

Disponível em: https://www.nucleodoconhecimento.com.br/saude/malaria-grave 


\section{RESULTADOS E DISCUSSÃO}

A Amazônia, região onde o estado do Amapá está localizado contribui para formação do elo epidemiológico de transmissão da malária devido suas características geográficas, climáticas e os aspectos socioculturais. Desse modo, o conhecimento da dinâmica a doença, na perspectiva da peculiaridade de cada região endêmica e de faixa etária de desenvolvimento populacional, é de grande importância. (BRASIL, 2016).

Inicialmente caracterizou-se os aspectos epidemiológicos dos pacientes em estudo, quanto ao gênero, faixa etária, procedência e espécie infectante. Dessa forma, observou-se predominância de pacientes do sexo masculino os quais totalizaram $63,8 \%(n=30)$ dos casos, enquanto somente $36,2 \%(n=17)$ pertenciam ao sexo feminino (Figura 1).

Figura 1 - Porcentagem de casos de malária grave em 2016 no estado do Amapá, por gênero.

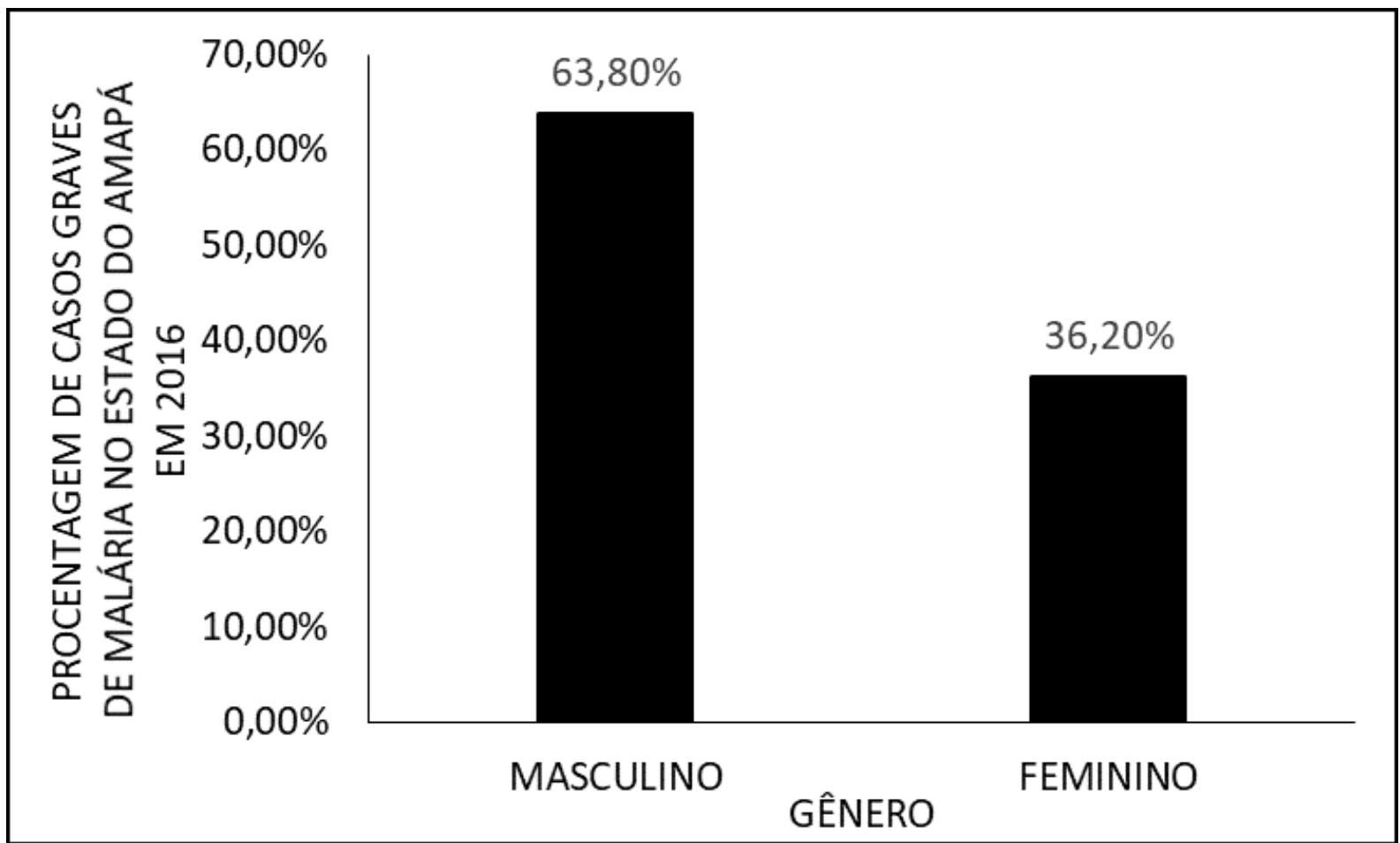

RC: 52768

Disponível em: https://www.nucleodoconhecimento.com.br/saude/malaria-grave 
Para Monteiro, Ribeiro e Fernandes (2013), estão sob risco de contrair malária indivíduos de ambos os sexos. Homens tendem a serem menos cuidadosos com a saúde do que as mulheres. Trabalhos em áreas propensas à presença de vetores também costumam ser ocupados por homens (ALMEIDA et al., 2020).

Mais da metade $28(59,6 \%)$ dos casos ocorreram em pacientes que pertenciam as faixas etárias até 5 anos; seguido de 6 a 10 anos, com 29,8\% $(n=14)$ dos casos. Apenas $10,6 \%(n=5)$ dos casos ocorreram nos pacientes de 11 a 15 anos (Figura 2).

Figura 2 - Porcentagem de casos de malária grave em 2016 no estado do Amapá, por faixa etária.

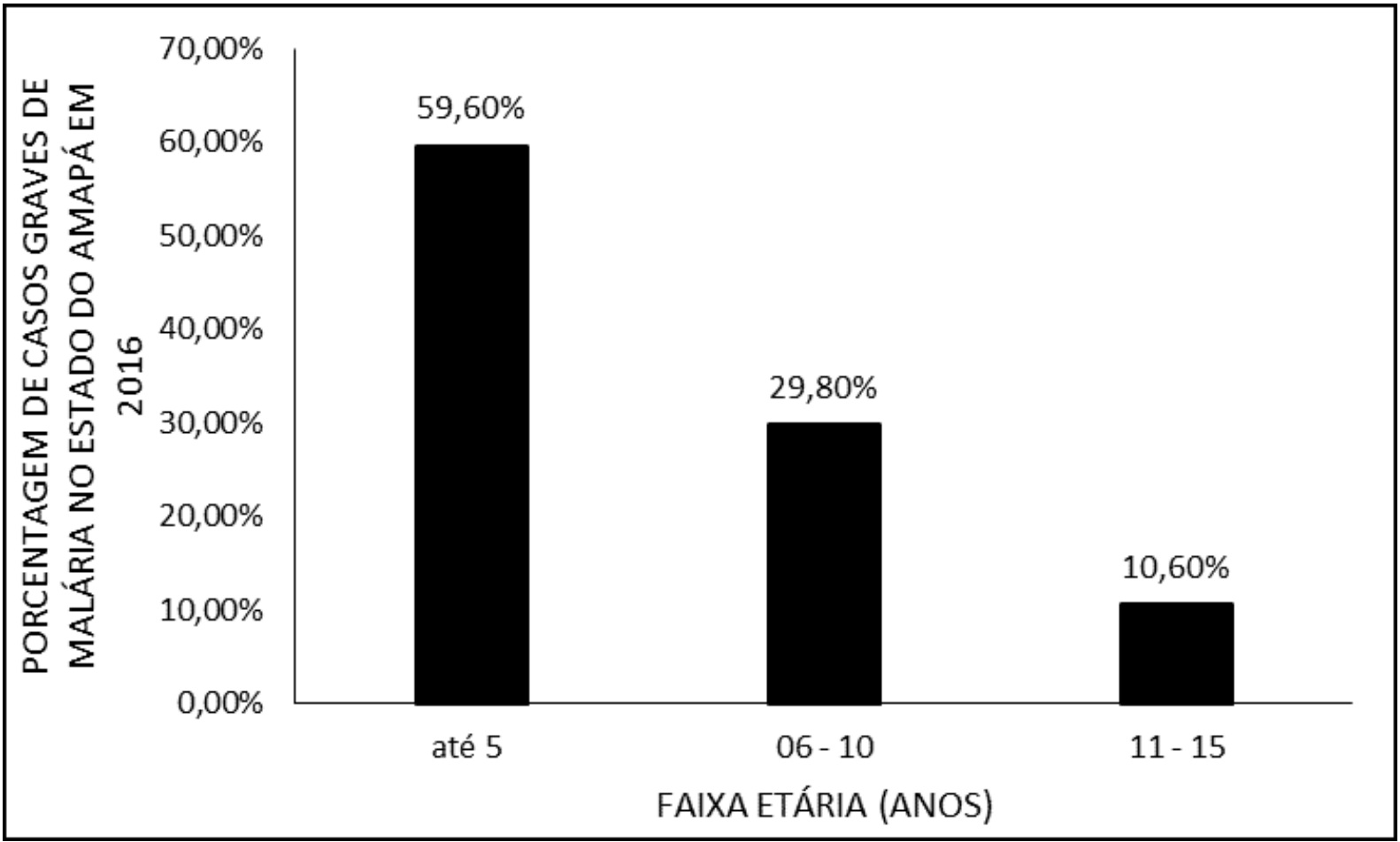

Em relação a faixa etária até 5 anos, em que os números de casos foram mais elevados, pode estar relacionado com a susceptibilidade da criança em adquirir doenças, também por fazerem parte do grupo de risco e o fato de nesse estudo prevalecer a primo-infecção $94 \%$ dos casos que é um fator que contribui para a

RC: 52768

Disponível em: https://www.nucleodoconhecimento.com.br/saude/malaria-grave 
gravidade já que o indivíduo não apresenta nenhuma imunidade específica contra o plasmódio (VENTURA et al., 1999; GOMES et al., 2011; RIBEIRO, 2012).

Quanto à espécie infectante, nesse estudo não houve registro nos prontuários da presença de $P$. malarie e nem malária mista, destacando-se, com maior frequência as infecções por Plasmodium vivax com $72 \%(n=34)$ em relação ao $P$. falciparum que envolveu $28 \%(n=13)$ dos casos registrados (Figura 3 ).

Figura 3 - Porcentagem de casos de malária grave em 2016 no estado do Amapá, por faixa etária.

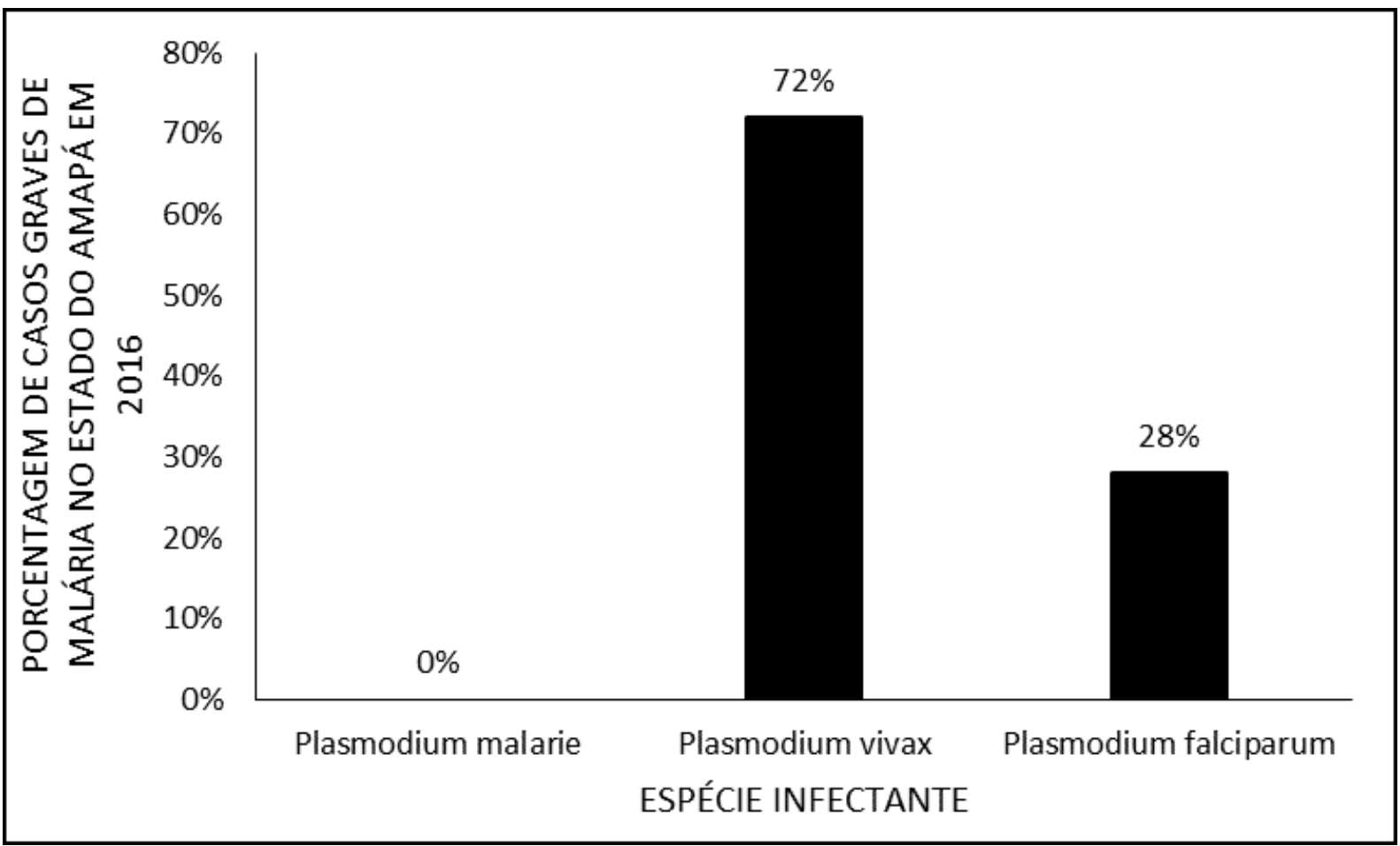

Este achado corrobora com o encontrado por Santos e Santos (2011), que em seu estudo evidenciam maior incidência de infecção por $P$. vivax no estado do Amapá. Por outro lado, quando se analisa pelo aspecto gravidade essa quantidade de infecção por $P$. vivax não é tão comum, já que a maioria dos levantamentos apresentam malária grave relacionada ao $P$. falciparum (KIRCHGATTER;

RC: 52768

Disponível em: https://www.nucleodoconhecimento.com.br/saude/malaria-grave 
PORTILLO, 2005; GOMES, 2011; MIOTO; GALHARDI; AMARANTE, 2012; PARISE, 2009).

A procedência desses pacientes conforme a tabela 1, mostra que entre os municípios amapaenses, Macapá se destacou com 29 casos, sendo 79\% $(\mathrm{n}=23) P$. vivax e $21 \%$ (6) $P$. falciparum. Dado que pode ser atribuído ao fato do trabalho ter sido realizado na Capital.

Tabela 1 - Distribuição dos pacientes internados no HCA com malária grave de acordo com o município de residência e espécie infectante em 2016, Macapá-AP.

\begin{tabular}{|c|c|c|c|c|c|c|}
\hline \multirow{2}{*}{ Município de procedência/ Espécie infectante } & \multicolumn{2}{|c|}{ P. vivax } & \multicolumn{2}{|c|}{ P. falciparum } & \multicolumn{2}{|c|}{ Total } \\
\hline & $\mathrm{N}$ & $\%$ & $\mathrm{~N}$ & $\%$ & $\mathrm{~N}$ & $\%$ \\
\hline Macapá & 23 & 79 & 6 & 21 & 29 & 100 \\
\hline Mazagão & 2 & 67 & 1 & 33 & 3 & 100 \\
\hline Ferreira Gomes & 1 & 25 & 3 & 75 & 4 & 100 \\
\hline Porto Grande & 1 & 33 & 2 & 67 & 3 & 100 \\
\hline Tartarugalzinho & 4 & 100 & 0 & 0 & 4 & 100 \\
\hline Oiapoque & 1 & 100 & 0 & 0 & 1 & 100 \\
\hline Outros Estados (PA) & 2 & 67 & 1 & 33 & 3 & 100 \\
\hline Total & 34 & 72 & 13 & 28 & 47 & 100 \\
\hline
\end{tabular}

Fonte: Same/HCA

Ocorrência semelhante foi observada em uma pesquisa, desenvolvida em um hospital de referência em Belém do Pará, em que também os demais municípios isoladamente foram pouco representativos (MONTEIRO; RIBEIRO; FERNANDES, 2013). Assim, embora o estado do Amapá inteiro, seja área endêmica, supõe-se que a população está sendo assistida, com diagnóstico e tratamento adequado nas unidades de menor complexidade nos municípios de residência.

Ainda sobre a tabela 1, a distribuição das espécies por município, difere dos demais apresentados, Ferreira Gomes com 75\% (3/4) e Porto Grande 67\% (2/3) de infecção por $P$. falciparum, é onde verifica-se um ligeiro predomínio dos casos de infecção por esta espécie, achado esse comum em vários estudos onde prevalece os casos

RC: 52768

Disponível em: https://www.nucleodoconhecimento.com.br/saude/malaria-grave 
de malária grave causado por P. falciparum (KIRCHGATTER; PORTILLO, 2005; PARISE, 2009; GOMES, 2011; MIOTO; GALHARDI; AMARANTE, 2012).

O município amapaense com menor número de caso foi Oiapoque com apenas 1 causado por $P$. vivax. O restante de 3 casos foi atendimento realizado em pacientes provenientes de outro estado, neste caso Pará das cidades de Breves e Afuá, sendo $67 \%(\mathrm{n}=2)$ P. vivax e 33\% (1) P. falciparum.

Os meses com maior frequência de internações por malária grave foram os meses de setembro e novembro com 8 (17\%) das internações cada um. Enquanto os meses de março, abril e maio foram os que apresentaram menor número apenas $2 \%$ (1) de internação conforme mostra a (figura 4). A sazonalidade da malária é variável em cada estado da região amazônica, porém em geral, há um pico de casos no período de transição entre as estações seca e úmida (BRASIL, 2016).

Figura 4 - Porcentagem de casos de malária grave em 2016 no estado do Amapá, por meses do ano.

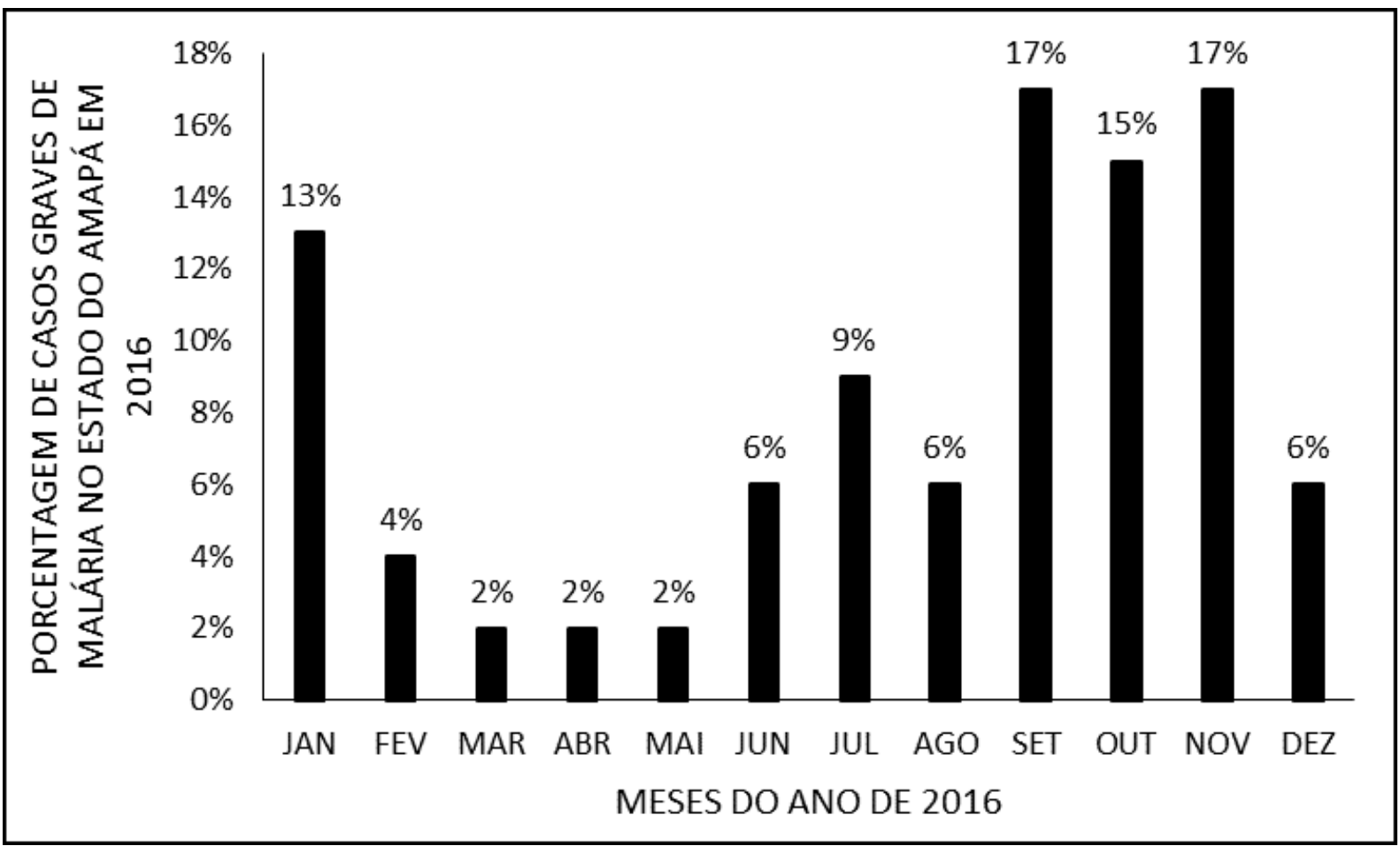

RC: 52768

Disponível em: https://www.nucleodoconhecimento.com.br/saude/malaria-grave 
O principal vetor da malária no Brasil, o Anopheles darlingi, reproduz-se em grande quantidade nas águas de baixo fluxo, profundas e sombreadas, e a sua reprodução diminui nos períodos chuvosos (MONTEIRO; RIBEIRO; FERNANDES, 2013). No estado do Amapá, o mês de setembro apresenta um baixo índice de chuva e novembro o início da estação de chuvas, fato que pode justificar o maior número de internação por malária grave neste estudo. Certamente, além das mudanças climáticas, outros fatores, como socioambientais e principalmente, variações na qualidade e quantidade das ações de controle podem estar relacionada à maior ou menor incidência da doença (BRASIL, 2016).

Quanto ao tempo de internação, vários fatores podem influenciar na permanência desses pacientes no ambiente hospitalar, a (figura 5) evidencia que a maioria dos pacientes permaneceram internados até 5 dias. Os dois pacientes infectados por $P$. vivax que permaneceram de 16 a 20 dias ambos apresentavam quadro de desidratação, anemia e plaquetopenia, sendo que um deles teve a mais, leucopenia, icterícia, colúria e resultados de exames das transaminases bastante elevados.

Figura 5 - Porcentagem de casos de malária grave em 2016 no estado do Amapá, por dias de internação.

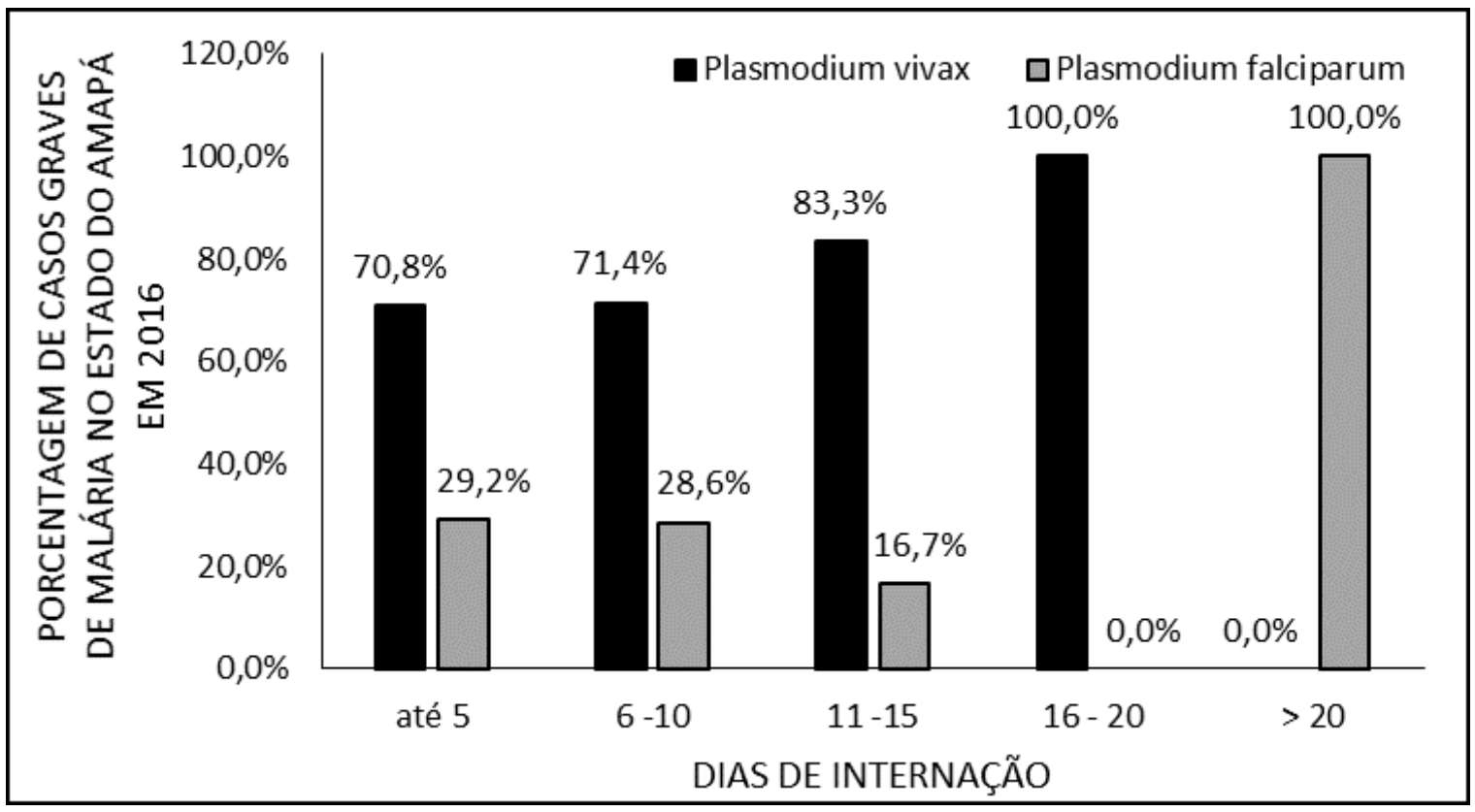

RC: 52768

Disponível em: https://www.nucleodoconhecimento.com.br/saude/malaria-grave 
Já o paciente com mais de 20 dias com infecção por $P$. falciparum, tinha um ano de idade, apresentou alta parasitemia de $192.000 \mathrm{f} / \mathrm{mm}^{3}$, hepatoesplenomeglia, plaquetopenia, anemia intensa e realizou transfusão de concentrado de hemácias. Tem sido bem documentado que a anemia intensa, uma manifestação clínica presente na malária grave e é um importante fator que contribui para a morbidade, tempo de internação e até mesmo a mortalidade, ocorrendo em infecções por $P$. falciparum e com bastante frequência também por P.vivax (PRICE et al., 2007; Naing et al., 2014).

De acordo com as informações analisadas, o tempo decorrido até o diagnóstico da malária que também é um fator decisivo para cura ou evolução para gravidade, foram: até 3 dias $40 \%$, de 4 a 5 dias $38 \%$ dos pacientes, de 6 a 10 dias $17 \%$ e de 11 a 15 dias $4 \%$ dos casos. Todos os pacientes só receberam alta após melhora do quadro clinico e laboratorial, com encaminhamento para controle ambulatorial com infecto pediatra, não houve registro de óbito no período estudado.

As manifestações clínicas encontradas com maior frequência foram: febre, palidez, tosse, distensão abdominal, dor abdominal, desidratação, cefaleia, calafrio e diarreia (tabela 2). A febre esteve presente em $100 \%$ dos casos, o que para Ramos (2006), é o principal motivo que leva a maioria dos pacientes a procura de cuidados médicos e a realização de exames complementares de diagnóstico.

A palidez por sua vez, ocorreu em $96 \%$ dos pacientes, pois é uma manifestação clínica muito frequente nos casos de malária, resultado semelhante com o de Ventura et al. (1999) que no seu estudo realizado também com crianças e adolescentes (85\%) apresentaram palidez.

Já o calafrio e a cefaléia ambos ocorreram em apenas 30\% (14/47) dos pacientes. São sintomas que na maioria das vezes acompanham a febre, formando a clássica tríade malárica (BRASIL, 2009). Talvez não tenha sido observado ou pela falta de registro no momento da anamnese, ou por se tratar de um estudo com casos graves onde as manifestações podem se apresentarem de forma atípica.

RC: 52768

Disponível em: https://www.nucleodoconhecimento.com.br/saude/malaria-grave 
A dor abdominal esteve presente em (32\%) e a diarreia $(23 \%)$ dos pacientes, segundo Ramos (2006), podem ter origem malárica, porém nas áreas endêmicas, geralmente as condições socioeconômicas são precárias, com saneamento inadequado o que pode levar a infecções por parasitas intestinais pela ingestão de alimentos e água contaminada, o que pode somar e intensificar esses sintomas no momento da infecção.

Os pacientes que apresentaram ascite, colúria, sonolência, e diarreia foram infectados por $P$. vivax. Já os que apresentaram esplenomegalia, anorexia e taquicardia tiveram infecção por $P$. falciparum, as outras manifestações da tabela 2 ocorreram tanto em infecções por $P$. falciparum quanto por $P$. vivax.

Tabela 2 - Distribuição das manifestações clínicas presentes em pacientes internados no HCA com malária grave no ano 2016. Macapá, Amapá.

\begin{tabular}{|c|c|c|}
\hline \multirow{2}{*}{ Manifestações Clínicas } & \multicolumn{2}{|c|}{ Frequencia $(\mathrm{N}=47)$} \\
\hline & $\mathrm{N}$ & $\%$ \\
\hline Febre & 47 & 100 \\
\hline Palidez & 45 & 96 \\
\hline Tosse & 20 & 43 \\
\hline Distensão Abdominal & 20 & 43 \\
\hline Dor Abdominal & 15 & 32 \\
\hline Desidratação & 14 & 30 \\
\hline Calafrio & 14 & 30 \\
\hline Cefaléia & 14 & 30 \\
\hline Diarreia & 11 & 23 \\
\hline Edema & 6 & 13 \\
\hline Náuseas & 6 & 13 \\
\hline Hepatomegalia & 5 & 11 \\
\hline Hematúria & 4 & 9 \\
\hline Colúria & 3 & 6 \\
\hline Ascite & 2 & 4 \\
\hline Sonolência & 2 & 4 \\
\hline Esplenomegalia & 1 & 2 \\
\hline Anorexia & 1 & 2 \\
\hline Taquicardia & 1 & 2 \\
\hline
\end{tabular}

Fonte: Same/HCA

RC: 52768

Disponível em: https://www.nucleodoconhecimento.com.br/saude/malaria-grave 
Recentemente, enfatiza-se a ocorrência de casos graves de malária por $P$. vivax (SIROMA, 2016; WASSMER et al., 2015; LACERDA et al., 2012). Muitas das manifestações clínicas de maior gravidade que ocorrem na malária por $P$. falciparum também têm sido descritas em pacientes com P. vivax (MONTEIRO; RIBEIRO; FERNANDES, 2013; SALAS et al., 2013). Fato evidenciado no presente estudo (tabela 3), pacientes diagnosticados com malária por $P$. vivax apresentaram vômitos, icterícia, dispinéia, anemia intensa, oligúria, convulsão e hemorragia. São condições que indicam gravidade e necessidade de internação (BRASIL, 2010).

A condição de gravidade mais frequente foi vômito, $100 \%$ nos pacientes infectados por $P$. Falciparum e $68 \%$ nos infectados por $P$. vivax, situação que nos prontuários justificaram boa parte das internações, pelo fato das crianças não tolerarem a medicação via oral, necessitando assim de medicação intravenosa. De acordo com Ramos (2006), vômito pode atrapalhar o tratamento correto, levando a não ingestão dos medicamentos ou absorção inadequada dos mesmos, além de levar a quadros de desidratação, como visto na (tabela 2) onde 30\% (14/47) apresentaram desidratação.

Sinais e sintomas respiratórios como tosse (tabela 2) e dispinéia (tabela 3) foram registrados tanto em pacientes infectados por $P$. falciparum como por $P$. vivax, porém não se pode afirmar que foram causados exclusivamente pela malária, pois não foram analisados os resultados de gasometria, espirometria e exames radiográficos da maioria, apenas esse último em um paciente que apresentou diagnóstico de malária associada a quadro de pneumonia.

Os sinais e sintomas respiratórios e complicações pulmonares, tem sido alvo de estudo. Val et al. (2017) evidenciaram que complicações respiratórias estiveram significantemente associadas a casos de pacientes com malária grave que foram a óbito. Carvalho et al. (2010) relatou que P. vivax tem a capacidade de aderência em células endoteliais pulmonares humanas. Para Salas et al. (2013) processos fisiopatogênicos poderiam ser compartilhados por essas duas espécies, como sequestro de eritrócitos infectados da circulação periférica para o interior de órgãos

RC: 52768

Disponível em: https://www.nucleodoconhecimento.com.br/saude/malaria-grave 
vitais (pulmão, cérebro e rins) e citoaderência à parede endotelial de capilares desses órgãos.

De acordo com Carvalho et al. (2010), essas observações levam a uma modificação dos paradigmas atuais da patogênese da malária e abre caminho para investigar a fisiopatologia das infecções por $P$. vivax, pois como os eritrócitos infectados por $P$. vivax não possuem estruturas de botão (modificações na superfície) e porque todas as formas do parasita podem ser observadas no sangue periférico dos pacientes, tornou-se dogma que $P$. vivax não tem capacidade de sequestrar e, portanto, nem de citoaderência (LOPES et al., 2014).

Outra condição de gravidade encontrada, foi a anemia intensa, em (5/47) casos, sendo 4 infecções por $P$. vivax e 1 por $P$. falciparum. Anemia grave que é definida como hematócrito menor que $15 \%$ e hemoglobina inferior a $5 \mathrm{~g} / \mathrm{dL}$, geralmente está presente na malária grave devido a intensa destruição e sequestro de hemácias, alteração na eritropoiese, e eventuais hemorragias (BRASIL, 2010; GOMES et al., 2011; SIROMA, 2016).

No presente estudo houve registro de nove pacientes que realizaram transfusão sanguínea, necessitaram de concentrado de hemácias, sendo que 2 dos 9 precisaram também de plaquetas, desses 9 pacientes oito foram infectados por $P$. vivax, e apenas um por $P$. falciparum. O paciente com hemorragia foi um dos pacientes que fez transfusão sanguínea de concentrado de hemácias e plaquetas.

Complicações semelhantes referente a mlária grave envolvendo $P$. vivax foram descritas em outros estudos como anemia grave, disfunção hepática, icterícia, trombocitopenia com ou sem sangramento (WASSMER et al., 2015; LACERDA et al., 2012; SALAS et al., 2013; )

Em relação a idade $<1$ ano em que houve (10/47) pacientes (tabela 3), para Gomes et al (2011), a gravidade da malária depende da relação entre hospedeiro (vulnerabilidade e estado imunológico) e o Plasmodium spp (espécie infectante e

RC: 52768

Disponível em: https://www.nucleodoconhecimento.com.br/saude/malaria-grave 
densidade parasitária). E as crianças pequenas, os primoinfectados e as gestantes são hospedeiros que se encontram mais vulneráveis às formas graves da doença, como já vem sendo mencionado no decorrer do trabalho.

Tabela 3 - Distribuição das condições que indicam gravidade e necessidade de internação de acordo com a espécie infectante em pacientes internados com malária grave no ano 2016, Macapá - AP.

\begin{tabular}{lccccccc}
\hline \multirow{2}{*}{\begin{tabular}{c} 
Condições que indicam gravidade $\begin{array}{c}\text { Espécie Infectante } \\
\text { necessidade de internação }\end{array}$ \\
\cline { 2 - 8 }
\end{tabular}} & $\begin{array}{c}\text { P. falciparun } \\
(\mathrm{N}=13)\end{array}$ & $\begin{array}{c}P . \text { Vivax } \\
(\mathrm{N}=34)\end{array}$ & \multicolumn{3}{c}{$\begin{array}{c}\text { Total } \\
(\mathrm{N}=47)\end{array}$} \\
\cline { 2 - 8 } & $\mathrm{N}$ & $\%$ & $\mathrm{~N}$ & $\%$ & $\mathrm{~N}$ & $\%$ \\
\hline Vômitos repetidos & 13 & 32 & 28 & 68 & 41 & 100 \\
Icterícia & 3 & 27 & 8 & 73 & 11 & 100 \\
Dispneia & 3 & 30 & 7 & 70 & 10 & 100 \\
Idade $<1$ ano & 0 & 0 & 10 & 100 & 10 & 100 \\
Anemia Intensa $(\mathrm{Hb}<5 \mathrm{~g} / \mathrm{dL})$ & 1 & 20 & 4 & 80 & 5 & 100 \\
Oligúria & 1 & 33 & 2 & 67 & 3 & 100 \\
Convulsão & 0 & 0 & 2 & 100 & 2 & 100 \\
Hemorragias & 0 & 0 & 1 & 100 & 1 & 100 \\
\hline
\end{tabular}

O nível de parasitemia mais elevado foi registrado na infecção por $P$. falciparum com $192.000 \mathrm{f} / \mathrm{mm}^{3}$. Entre os pacientes estudados, $P$. vivax evidenciou nível máximo de $30.000 \mathrm{v} / \mathrm{mm}^{3}$. Os principais eventos patogênicos atribuídos ao $P$. falciprum são bem descrito na literatura, como capacidade de infectar eritrócitos em todos os estágios, apresentando assim altas parasitemias, o fenômeno do sequestro de eritrócitos infectados da circulação sanguínea periférica e a capacidade de adesão ao endotélio de capilares de órgãos internos, de modo que poucas formas maduras são observadas em amostras de sangue periférico durante a infecção (KIRCHGATTER; PORTILLO, 2005; CARVALHO et al., 2010).

Quanto ao perfil laboratorial (tabela 4), os exames hematológicos mostraram que 91\% (43/47) dos pacientes apresentaram hemácias abaixo dos valores de referência e $100 \%$ dos casos estavam com hemoglobina e hematócrito baixos. Apresentaram plaquetopenia (número de plaquetas reduzido) $n=34$ pacientes, entre esses, $71 \%$

RC: 52768

Disponível em: https://www.nucleodoconhecimento.com.br/saude/malaria-grave 
( $n=24)$ tiveram malária por $P$. vivax e $29 \%(n=10) P$. falciparum. Essas evidências hematológicas são muito frequentes, principalmente nos casos de malária grave (RAMOS, 2006; LACERDA et al., 2012; SIROMA, 2016).

Tabela 4 Distribuição dos resultados dos exames laboratoriais dos pacientes atendidos com malária grave no ano 2016, Macapá - AP.

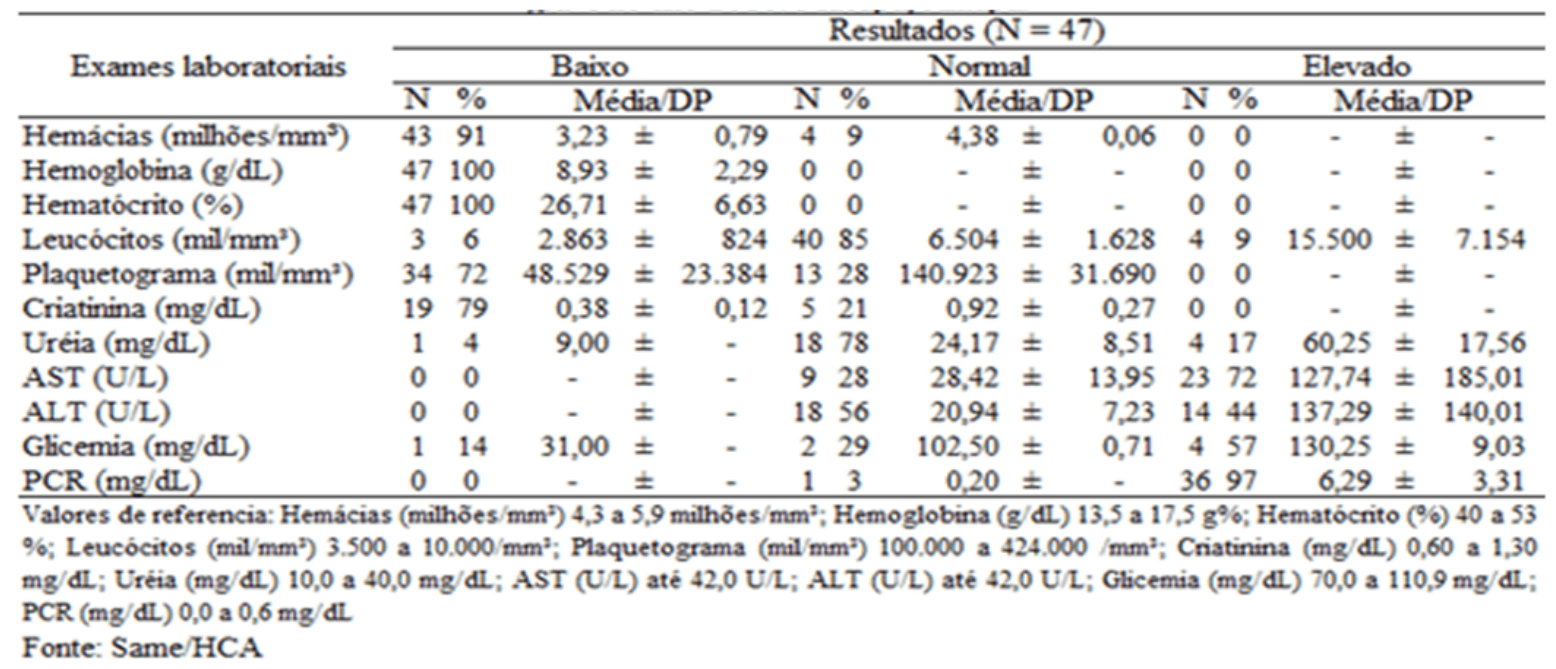

Em relação as dosagens de uréia e creatinina que avaliam as funções renais, apenas uréia se destacou com valores elevados, que pode indicar comprometimento dos rins. Dos (23/47) pacientes que realizaram o referido exame, apenas $17 \%(n=4)$ apresentaram valores elevados, sendo todos acometidos por $P$. vivax.

Já os exames que fazem parte da avaliação hepática, o aspartato aminotransferase (AST) e a Alanina aminotransferase (ALT) ambos foram realizados em 68\% (32/47) dos pacientes, sendo que dos 32 , apresentaram valores elevados de (AST) $72 \%$ $(\mathrm{n}=23)$, entre os quais $22 \%(\mathrm{n}=7)$ eram $P$. falciparum e $50 \%(\mathrm{n}=16)$ eram P.vivax. $\mathrm{E}$ $44 \%(14 / 32)$ estavam com resultados elevados de (ALT), sendo 19\% $(n=6)$ eram $P$. falciparum e $25 \%(n=8)$ eram $P$. vivax.

A elevação das transaminases (tabela 4) assim como a presença da icterícia (tabela 3) evidenciam comprometimento hepático, como o ciclo assexuado do plasmódio

$\mathrm{RC}: 52768$

Disponível em: https://www.nucleodoconhecimento.com.br/saude/malaria-grave 
chamado pré-eritrocítico ou esquizogonia tecidual, ocorre no fígado rompendo os hepatócitos, acaba levando a um processo inflamatório nesse órgão (RAMOS, 2006; BRASIL, 2009).

Quanto ao teste de glicemia foi detectado que apenas 7/47 (15\%) foram realizados, onde desse total $(\mathrm{n}=1)$ acometido por $P$. Vivax, apresentou hipoglicemia que é uma ocorrência comum na malária grave, ocorre geralmente em associação com outras complicações (GOMES, 2011; RIBEIRO, 2012).

Chama a atenção o fato de que 40/47 (85\%) dos pacientes não realizaram teste glicêmico, segundo o Guia Prático de Tratamento de Malária no Brasil, glicemia é uma das determinações laboratoriais, assim como hemograma, determinação da parasitemia, gasometria arterial e parâmetros de função renal e hepática (BRASIL, 2010). A falta de documentação de resultados de exames dos pacientes dificulta a observação da dinâmica a doença, ou seja, traçar o perfil clínico dos pacientes.

A dosagem de Proteína C reativa (PCR) foi realizada em 79\% (37/47) dos pacientes, sendo que desses $(n=37), 97 \%(n=36)$ apresentaram níveis elevados de proteína C reativa. Os pacientes com níveis elevados foram $24 \%$ ( $n=9)$ P. falciparum e $73 \%$ (n=27) $P$. vivax.

A Proteína $C$ reativa é sintetizada no fígado é um marcador não específico que se eleva em respostas de fase aguda quando há estímulo de lesão tecidual, inclusive em infecções por diversos microrganismos (COLLARES, 2006). Evidências já foram identificadas sobre o aumento dos níveis no plasma de proteína $C$ reativa e a progressão de gravidade da malária (LACERDA et al., 2012).

$P$. vivax e $P$. falciparum constituem as principais espécies causadoras de malária humana, por serem responsáveis pela maioria dos casos de morbidade e mortalidade no globo. Enquanto $P$. falciparum é considerada a espécie mais letal, $P$. vivax é considerada a mais amplamente distribuída no globo (BATTLE et al., 2014).

RC: 52768

Disponível em: https://www.nucleodoconhecimento.com.br/saude/malaria-grave 


\section{CONCLUSÕES}

Esse estudo evidenciou o perfil epidemiológico, clínico e laboratorial da malária grave em crianças e adolescentes, levando consideração não somente as infecções por Plasmodium falciparum que comumente é preconizado como o principal causador da malária grave, mas foram cuidadosamente descritas as complicações relacionadas ao Plasmodium vivax.

Foi possível observar dentro do presente estudo tanto o $P$. falciparum como $P$. vivax associados a quadros de malária grave. Encontrou-se uma proporção elevada de infecção $P$. vivax, a qual foi responsável pelo único caso de hemorragia, pelos dois únicos casos de convulsão e por oito transfusões sanguíneas. O encontro de muitas internações pela infecção por $P$. vivax ressalta o potencial de gravidade desta espécie.

Porém mais estudos são necessários para esclarecer os mecanismos fisiopatológicos que levam à malária grave por $P$. vivax. Uma vez que o novo cenário da malária evidencia que Plasmodium vivax vem modificando sua maneira de interagir com o hospedeiro humano, não podendo mais ser considerado um parasita benigno. A constatação dessa mudança de paradigma aumenta ainda mais os desafios no controle dessa doença, em vista de $P$. vivax ser considerada a espécie mais prevalente no mundo, isto poderá repercutir num agravamento da situação atual da malária.

Mesmo o presente estudo não traduzindo a prevalência populacional do Amapá, os dados aqui apresentados podem contribuir para orientar as demandas assistenciais, favorecer melhores adequações dos serviços especializados. Pois a identificação de dados epidemiológicos, clínicos e laboratoriais da malária grave se constituem numa estratégia para o diagnóstico precoce e tratamento adequado, visando mitigar os impactos causados pela doença.

RC: 52768

Disponível em: https://www.nucleodoconhecimento.com.br/saude/malaria-grave 


\section{REFERÊNCIAS}

ALMEIDA, H.K. S. et al. Casos confirmados de malária no Brasil entre os anos de 2011 e 2015. Revista Científica Multidisciplinar Núcleo do Conhecimento. Ano 05, Ed. 04, Vol. 07, pp. 05-16, 2020. ISSN: 2448-0959, Disponível em: $<$ https://www.nucleodoconhecimento.com.br/saude/malaria-no-brasil>, $\quad$ DOI: 10.32749/nucleodoconhecimento.com.br/saude/malaria-no-brasil

BATTLE, K. E. et al. Geographical variation in Plasmodium vivax relapse. Malaria Journal, 2014, 13:144. https://doi.org/10.1186/1475-2875-13-144.

BOTELHO, C. et al. Manifestações respiratórias na malária por Plamodium falciparum e vivax. Rev. Inst. Med. trop. São Paulo, 1987 29(6):337-345.

BRASIL. Revista Pan-Amazônica de Saúde, v.4, n.2, p.33-43, 2013. Disponível em: <http://dx.doi.org/10.5123/S2176-62232013000200005

BRASIL. Ministério da Saúde. Guia prático de tratamento da malária no Brasil. Brasília: Ministério da Saúde, 2010.

BRASIL. Ministério da Saúde. Secretaria de Vigilância em Saúde. Plano de eliminação de malária no Brasil. Brasília: Ministério da Saúde, 2016.

BRASIL. Ministério da Saúde. Conselho Nacional de Saúde. Resolução № 466, de 12 de dezembro de 2012. Diretrizes e normas regulamentadoras de pesquisas envolvendo seres humanos. Diário Oficial da União, Brasília, DF, 13 jun. 2013. Seção I, p.59-62.

. Ministério da Saúde. Manual de diagnóstico laboratorial da malária. 2 ed. Brasília: Ministério da Saúde, 2009.

CARDOSO, Rosilene Ferreira. Efeito da sazonalidade na curva endêmica da malária por Plasmodium falciparum e vivax no garimpo do Lourenço: uma série temporal

RC: 52768

Disponível em: https://www.nucleodoconhecimento.com.br/saude/malaria-grave 
histórica na Zona da Amazônia Brasileira. UFRGS. 2014. Disponível em: http://hdl.handle.net/ 10183/114973. Acesso em: 06/11/2017.

CARVALHO, Bruna O. et al. On the Cytoadhesion of Plasmodium vivax-Infected Erythrocytes, The Journal of Infectious Diseases, Volume 202, Issue 4, 15 August 2010, Pages 638-647, https://doi.org/10.1086/654815. Acess: 12/09/2017.

COLLARES, Guilherme Birchal; PAULINO, Urquiza Helena Meira. Aplicações clínicas atuais da proteína c reativa. Rev Med Minas Gerais, 2006; 16(4): 227-333. Disponível em: http://rmmg.org/artigo/detalhes/579. Acesso em: 15/11/2017.

GOMES, Andréia Patricia. et al. Malária grave por Plasmodium falciparum. Rev Ter Intensiva. Viçosa (MG). v 23, n 358 - 369, 2011.

HADDAD, Nagib. Metodologia de estudos em ciências da saúde. São Paulo: Roca; 2004.

KIRCHGATTER, Karin; PORTILO, Hernando A. Del. Aspectos Clínicos e Moleculares da Malária Grave. Anais da Academia Brasileira de Ciências. Rio de Janeiro, v. 77, n 3, 2005.

LACERDA, M. V. et al. Understanding the clinical spectrum of complicated Plasmodium vivax malaria: a systematic review on the contributions of the Brazilian literature. Malaria Journal, 11, 12.2012.

LOPES, Stefanie C. P. et al. Paucity of Plasmodium vivaxMature Schizonts in Peripheral Blood Is Associated With Their Increased Cytoadhesive Potential, The Journal of Infectious Diseases, Volume 209, Issue 9, 1 May 2014, Pages 14031407, https://doi.org/10.1093/infdis/jiu018. Acess: 12/09/2017.

MACIEL, Giovana Belem Moreira Lima; OLIVEIRA, Elaine Cristina de. Perfil entomológico e epidemiológico da malária em região garimpeira no norte do Mato Grosso, 2011. Epidemiol. Serv. Saúde, Brasília, v. 23, n. 2, p. 355-360, jun. 2014.

$\mathrm{RC}: 52768$

Disponível em: https://www.nucleodoconhecimento.com.br/saude/malaria-grave 
MIOTO, Leide Daiana; GALHARDI, Ligia Carla Faccin; AMARANTE, Marla Karine. Aspectos parasitológicos e imunológicos da malária. Biosaúde, Londrina, v. 14, n. 1, 2012. Disponível: http://www.uel.br/revistas/uel/index.php/biosaude/article/view/ 24324/17894. Acesso em: 07/11/2017.

MONTEIRO, M. R. C. C.; RIBEIRO, M. C.; FERNANDES, S. C. Aspectos clínicos e epidemiológicos da malária em um hospital universitário de Belém, Estado do Pará,

NAING, Cho et al. "Is Plasmodium Vivax Malaria a Severe Malaria?: A Systematic Review and Meta-Analysis." PLoS Neglected Tropical Diseases 8.8 (2014): e3071. PMC. Web. 16 Nov. 2017. Doi: 10.1371/journal.pntd.0003071

PARISE, Éldi Vendrame. Malária grave em Palmas, Estado do Tocantins: relato de caso. Rev. Soc. Bras. Med. Trop. [online]. 2009, vol.42, n.4, pp.463-468. ISSN 0037-8682. http://dx.doi.org/10.1590/S0037-86822009000400021.

PRICE, R. N. et al. Vivax malaria: neglected and not benign. The American Journal of Tropical Medicine and Hygiene, Volume 77, Issue 6_Suppl, Dec 2007, p. 79 87. DOI: https://doi.org/10.4269/ajtmh.2007.77.79

RAMASAMY, Ranjan. Zoonotic malaria-global overview and research and policy needs. Frontiers in public health, v. 2, 2014.

RAMOS, Cristina Bastos Silva Raposo. Malária vivax na pré-amazônia maranhense: aspectos epidemiológicos e características clínicas. 2006. 110 f., il. Dissertação (Mestrado em Ciências Florestais)-Universidade de Brasília, Brasília, 2006.

SALAS, Briegel de Las et al. Adherence to human lung microvascular endothelial cells (HMVEC-L) of Plasmodium vivax isolates from Colombia. Malaria Journal. 2013,12: 347-10.1186/1475-2875-12-347. https://doi.org/10.1186/1475-2875-12-347. Access: $12 / 11 / 2017$.

RC: 52768

Disponível em: https://www.nucleodoconhecimento.com.br/saude/malaria-grave 
SANTOS, Rafael Cleison Silva dos; SANTOS, Elinaldo da Conceição dos. Malária: cobaias humanas no Amapá. Estação Científica (UNIFAP). Macapá, v. 1, n. 2, p. 143-150, 2011. Disponível em: https://periodicos.unifap.br/index.php/estacao/article/ download/ 244/cleisonv1n2.pdf. Acesso : 08/10/2016.

SIROMA, Thais Keiko; FERRARI, Eduardo Cal; RIGO, Rosângela Silva. Plasmodium vivax: causa de malária grave. Revista da Sociedade Brasileira de Clínica Médica. $2016 \quad$ jul-set;14(3):166-71. Disponível: http://www.sbcm.org.br/revistas/RBCM/RBCM-2016-03.pdf\#page $=47$

TA, Thuy H. et al. First case of a naturally acquired human infection with Plasmodium cynomolgi. Malaria journal, v. 13, n. 1, p. 68, 2014.

VAL, Fernando et al. Are respiratory complications of Plasmodium vivax malaria na underestimated problem?. Malaria Journal, v. 16, n. 1, p. 495, 2017.

VENTURA, Ana Maria Revorêdo da Silva, Et al. Malária por Plasmodium vivax em crianças e adolescentes - aspectos epidemiológicos, clínicos e laboratoriais. J Pediatr 75: 187-194, 1999.

WASSMER, Samuel C. et al. "Investigating the Pathogenesis of Severe Malaria: A Multidisciplinary and Cross-Geographical Approach." The American Journal of Tropical Medicine and Hygiene 93.3 Suppl (2015): 42-56. PMC. http://doi.org/10.4269/ajtmh.14-0841 Web. 19 June 2017.

Enviado: Junho, 2020.

Aprovado: Junho, 2020.

RC: 52768

Disponível em: https://www.nucleodoconhecimento.com.br/saude/malaria-grave 